Research Article

\title{
Clinical Trial Analysis of Different Stages of HBV Patients Treated with Human CIK Cells
}

Fangfang Xia ${ }^{1}$, Ruihua Song ${ }^{2}$, Jingjing Zhang ${ }^{1}$, Yang Zhu², Yao Yang ${ }^{1}$, Ling $\mathrm{He}^{1}$, Yun Cao ${ }^{1}$, Chenlu $\mathrm{Li}^{1}$, Zheng Wang ${ }^{1}$, Shangli Cheng ${ }^{1}$, Jian $\mathrm{Ni}^{1}$, Lijun $\mathrm{Ma}^{3}$, Ding $\mathrm{Li}^{2}$, Daxiang Cui

${ }^{1}$ Institute of Nano Biomedicine and Engineering, Shanghai Engineering Research Center for Intelligent Diagnosis and Therapy Instrument, Department of Instrument Science and Engineering, School of Electronic Information and Electrical Engineering; National Center for Translational Medicine, Collaborative Innovational Center for System Biology, Shanghai Jiao Tong University, 800 Dongchuan Road, Shanghai 200240, P. R. China.

${ }^{2}$ Center for Biotechnological Diagnosis and Therapy, The 261 Hospital of PLA, Beijing 100094, China.

${ }^{3}$ Department of Oncology, Shanghai Tongren Hospital, Shanghai JiaoTong University School of Medicine, 1111 Xianxia Road, Shanghai 200336, P. R. China.

Corresponding authors. E-mail: liding@126.com; ljma@163.com; dxcui@sjtu.edu.cn

Received: Sep. 21, 2016; Accepted: Sep. 28, 2016; Published: Sep. 29, 2016.

Citation: Fangfang Xia, Ruihua Song, Jingjing Zhang, Yang Zhu, Yao Yang, Ling He, Yun Cao, Chenlu Li, Zheng Wang, Shangli Cheng, Jian Ni, Lijun Ma, Ding Li and Daxiang Cui, Clinical Trial Analysis of Different Stages of HBV Patients Treated with Human CIK Cells. Nano Biomed. Eng., 2016, 8(3): 203-2II.

DOI: $10.5101 /$ nbe.v8i3.p203-211.

\begin{abstract}
Cellular immunotherapy has become a potential therapeutic method for different diseases. Herein, we reported clinical trial results of Cytokine-induced killer (CIK) cells used for patients with hepatitis B, cirrhosis and liver cancers from 2000 to 2015. Results showed CIK cell therapeutic effects were closely positively associated with CIK cell numbers, treated times and HBV genotypes. Different stages of $\mathrm{HBV}$ patients treated with $>10^{10} \mathrm{CIK}$ cells per time for more than ten times exhibited remarkable decrease of HBV DNA numbers $(P<0.01)$, ALT and AST gradually recovered to normal scope, cytokine factors such as IFN- $\gamma$, IL-1b, IL-2, IL-4, IL-6, IL-10, IL-22 and IL-27 exhibited obvious increase, lifespan of patients with cirrhosis and hepatocellular carcinoma were extended, and that all the patients felt better in sleep, diet and pain during the period of CIK therapy. In conclusion, CIK cell therapy is a good alternative therapeutic method and can be effectively used for treatment of different stages of HBV patients.
\end{abstract}

Keywords: Hepatitis B virus; Cirrhosis; Hepatocellular carcinoma; Cytokine-induced killer cells; Cell therapy; Cytokine factors

\section{Introduction}

Hepatitis B virus has become the main cause of hepatitis, cirrhosis and hepatocellular cancer in China. There are almost 0.12 billion patiants with HBV in China; the number of patients with chronic hepatitis $\mathrm{B}$ reaches almost over 20 million. Every year, there are almost 0.5 million new hepatitis $B$ patients, and nearly 0.33 million patients die of HBV in China. HBV patients were genotyped into $\mathrm{A}, \mathrm{B}, \mathrm{C}$, and $\mathrm{D}$ genotypes. Therefore, how to enhance the therapeutic effects of different stages of HBV patients has become the focus of treatment [1].

It is well known that patients with hepatitis B 
virus are very difficult to be cured completely, which is mainly associated with Chronic hepatitis B virus (HBV) persistent replication and high plasma HBV DNA concentrations in patients [1]. The leading cause of primary hepatic cellular carcinoma is HBV infection, and it has been a serious problem worldwide $[2,3]$. The surrogate marker of hepatitis $B$ virus DNA is hepatitis B e antigen ( $\mathrm{HBeAg})$. Cirrhosis and hepatocellular carcinoma (HCC) are mostly caused by HBV infection and are the third-most common cause of cancer death in the world according to the World Health Organization (2011) [3, 4]. It is estimated that 350-400 million people are chronic carriers of $\mathrm{HBV}$ in the world, the majority of whom are in Asia $(75 \%)[3,5,6]$. HBV infection varies geographically. Most of the Southeast Asia and Southern Europe patients acquired HBV in the perinatal period or early childhood. In contrast, the U. S. patients acquired it much later in life [7, 8]. Additionally, many factors are responsible for the hepatitis $\mathrm{B}$ virus infection, such as viral factors, host factors, ages at infection, etc. [5].

Due to the fact that HBV has stable nuclear covalently circular DNA (cccDNA), it cannot be efficiently cleared away by current antiviral agents. Interferon- $\alpha$ can eliminate HBV through specific cccDNA degradation but is associated with systemic side effects [9]. Also, RNA interference (RNAi)based therapeutics have been used to knock down viral RNAs expression so as to reduce the viral load as well as the viral proteins that lead to disease and influence the ability of the immune system to clear the virus [10]. Gordon et al. reported that of their study subjects, $2.5 \%$ patients among the untreated ones developed HCC, and that $2.4 \%$ patients developed HCC though they have received antiviral HBV treatment [11]. To suppress HBV load, long-term use of antiviral agents is required. The innate immune system stimulation could enhance its virus control ability and long-lasting effects. GS9620 is a potent and selective agonist of Toll-like receptor 7; it was used for chimpanzees treatment who has been infected with chronic HBV. After a period of treatment, HBV surface antigen and HBV e antigen of the serum decreased. The GS-9620 eliminates the HBV through activating Toll-like receptor 7 signaling pathways in immune cells of chimpanzees, which highly suggest that human immune system can be regulated for hepatitis B virus treatment [12].

Chronic HBV treatment-induced clearance is relevant to host immune responses [13]. The interplay between host immune response and virus replication results in the liver damage and viral control as well as clinical outcome [14]. Over the past two decades, more and more attempts have been made to treat cancer patients by immunotherapy $[15,16]$. Cancer immunotherapy might harness the immune system to attack tumors [17]. Therefore, immunotherapy is a promising and effective treatment option for patients with cancers. Compared to standard lymphokine-activated killer (LAK) cells, Cytokineinduced killer (CIK) cells-based therapies as adoptive immunotherapy possess enhanced antitumor cytotoxic activity because they can systemically traffic to solid tumors, as well as residual disease [18-20].

Cytokine-induced killer (CIK) cells are a subset of natural killer $\mathrm{T}$ lymphocytes that share some phenotypical and functional similarities. The CIK cells can be generated in large numbers from peripheral blood mononuclear cells (PBMC) ex-vivo with the timed addition of IFN- $\gamma$, monoclonal antibody (mAb) against CD3 (OKT3) and recombinant human Interleukin (IL)-2 [21]. Mature CIK cells present $\mathrm{CD}^{+} \mathrm{CD} 56^{+}$phenotype. $\mathrm{CD} 3^{+} \mathrm{CD} 56^{+}$phenotype is the main effective cellular subtype for killing tumor cells. Therefore, CIK cells have an extraordinary cytolytic potential to recognize autologous malignant cells and possess higher lytic activity against target tumor cells [18, 22-25]. CIK-mediated cellular lysis is major histocompatibility antigen (MHC) unrestricted cytotoxicity. The exact mechanism involved in recognizing and killing tumor cells is not completely known, but the interaction between NKG2D molecules (receptor) expressed on the CIK cells membrane and MIC A/B or ULBPs molecules (MHC ligands) on tumor cells seem to play the main roles [26-28]. NKG2D participates in the TCR-independent tumor cell recognition and killing [29].

In this clinical trial, we fully used the advantages of human CIK cells, and treated 1,480 patients including 1,000 hepatitis B virus patients, 400 cirrhosis patients and 80 liver cancer patients from clinical hospitals. The alanine aminotransferase (ALT), Aspartate transaminase (AST), HBV DNA, certain cytokines and lifespan were used as key markers to evaluate the CIK therapeutic effects. Our clinical trial results fully suggest that CIK cell therapy has great potential in treatment of different stages of patients with hepatitis $\mathrm{B}$ virus, cirrhosis and liver cancer. 


\section{Material and Methods}

\section{Preparation and characterization of CIK cells}

Peripheral blood mononuclear cells (PBMC) were obtained from patients (or lineal relatives) by density gradient centrifugation using lymphocyte separation medium (TBD science, China) under GMP conditions. PBMC were suspended in X-vivo 15 medium (Lonza, USA) at a concentration of $1 \times 10^{6} / \mathrm{mL}$ and incubated $24 \mathrm{~h}$ with $5 \% \mathrm{CO}_{2}$ at $37{ }^{\circ} \mathrm{C}$. 2,000 U/mL IFN- $\gamma$ (PeproTech, USA) was added to the medium at day; $0.500 \mathrm{ng} / \mathrm{ml}$ Mab-CD3 ( PeproTech, USA ), and 1,000 U/ml of recombinant human Interleukin (IL)-2 ( PeproTech, USA ) were added the next day. Fresh X-vivo 15 medium and 1,000 U/ml IL-2 were then added every three days. On day 14, the phenotype of CIK cells were analyzed by flow cytometry with FITC-conjugated anti-CD3 and PE-conjugated anti-CD56 (Becton, Dickinson and Company, USA).

\section{Clinical patient selection for clinical trials}

Our clinical trial studies were approved the Ethical Committee of the 261 hospital of PLA, and Tong Ren hospital. The selection standards for patients with hepatitis B for CIK treatment were as follows: All patients with hepatitis $B$ were identified with HBV DNA examination and treated with regular clinical drugs for more than 2 years; their therapeutic effects were bad, or appeared multi-drug resistance. These patients with hepatitis $B$ treated with regular interferon and anti-HBV drugs were selected as control patients. All cirrhosis patients were from hepatitis B virus patients treated with regular clinical drugs for more than five years, and were identified with B ultrasound and ELISA examination. These cirrhosis patients with hepatitis $B$ treated with regular interferon and antiHBV drugs were selected as control patients. All liver cancer patients were from cirrhosis patients with hepatitis B virus treated for more than ten years, and were identified with ultrasound imaging, CT imaging and AFP biomarker detection. These liver cancer patients with hepatitis $B$ treated with regular drugs were selected as control patients. Finally, we selected 1,000 hepatitis B virus patients, 400 cirrhosis patients and 80 liver cancer patients in this clinical trial. Control patients included $100 \mathrm{HBV}$ patients treated with regular drugs, 100 cirrhosis patients treated with regular drugs and 20 hepatic cellular cancer patients treated with regular anti-cancer drugs. All patients were from 35 years to 65 years, male to female ratio was 2:1.
No other serious diseases existed in the selected trial patients.

\section{HBV DNA detection $\square$ genotyping analysis and biochemical analysis}

Serum HBV DNA was detected by real-time polymerase chain reaction during the study period (Roche Diagnostic Systems, Branchburg, NJ, USA), and then was further genotyping according to the previous report method [28, 29]. ALT, AST and AFP were determined by means of a kinetic UV test using a biochemical autoanalyser for clinic analyzers (Hitachi 736-15, Tokyo, Japan). Elevated ALT level was determined as ALT $\geq 40 \mathrm{U} / \mathrm{L}[30]$. The normal AST value was $8 \sim 28 \mathrm{U} / \mathrm{L}$ according to the suggestion of the Blood Center.

\section{Serum cytokine factor analysis}

The patient sera were collected before and after CIK treatment and sent for commercial service company to detect the concentration of cytokine factors such as GM-CSF, IFN-g, IL-1b, IL-13,IL-2, IL-4, IL-5, IL-6, TNF-a,IL-10, IL-22, IL-27 and AFP.

\section{Statistical analysis}

The paired student test was used in comparing liver indexes after CIK therapy. The Pearson correlation coefficient was calculated:

$\rho(X, Y)=\operatorname{cov}(X, Y) /\left(\sigma_{-} X \sigma_{-} Y\right)$,

where $\mathrm{X}$ is the population before the CIK therapy, $\mathrm{Y}$ is the population after the CIK therapy, cov is the covariance, and $\sigma$ is the standard deviation.

Statistical significance of obtained results was determined employing t-test and analysis of variance (ANOVA) using SPSS 18.0 software with $\mathrm{P}<0.05$ as a minimal level of significance.

\section{Results and Discussion}

\section{Phenotypic features of prepared CIK cells}

CIK cells were cultured according to the previous standard protocol [21]. The final immunophenotypes of CIK cells were shown in Fig. 1. The expression of CD3 and CD56 in CIK cells were $99.6 \%$ and $90.7 \%$, respectively. The CIK cells that co-express CD 3 and CD 56 molecules were about $85.1 \%$. All of these showed that CIK cells were successfully induced. Subsequently, we tested the bacteria, fungi and 

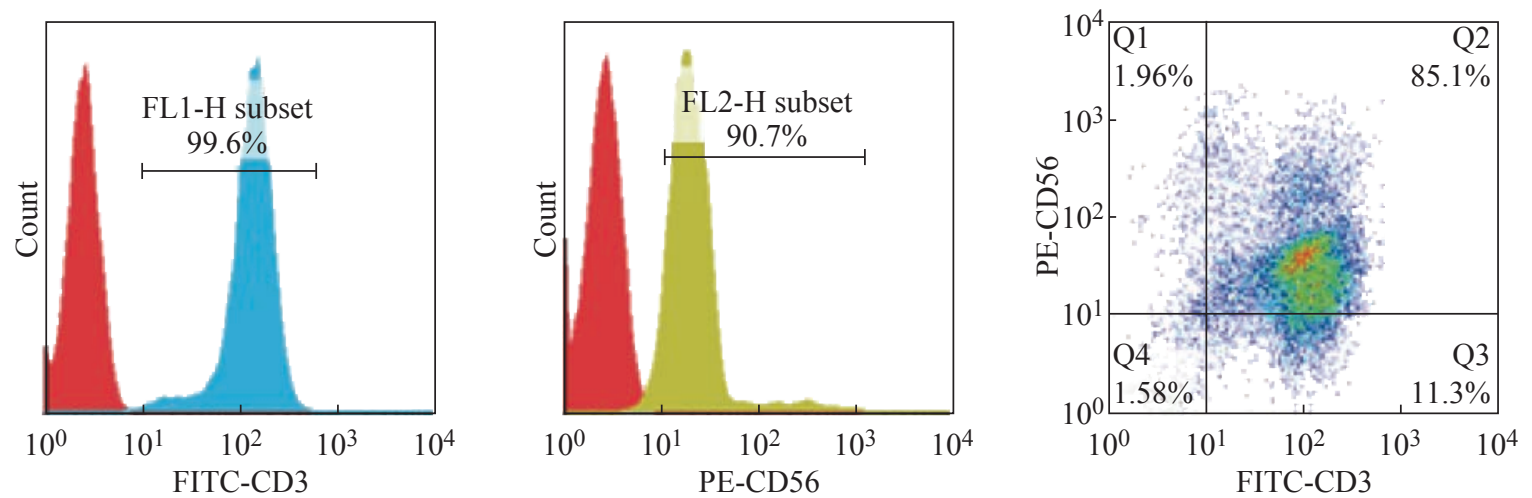

Fig. 1 Phenotype analysis of prepared CIK cells by flow cytometry (a) The expression of CD3 in CIK cells were 99.6\%; (b) The expression of CD56 in CIK cells were $90.7 \%$; (c) co-expression of CD3 and CD56 in CIK cells were 85.1\%.

Table 1 Genotypes of HBV in clinical trial patients

\begin{tabular}{|c|c|c|c|}
\hline Genotyping & Hepatitis patients & Cirrhosis patients & Liver cancer \\
\hline A & 150 & 60 & 20 \\
\hline B & 350 & 140 & 30 \\
\hline $\mathrm{C}$ & 410 & 152 & 26 \\
\hline D & 90 & 48 & 4 \\
\hline
\end{tabular}

endotoxin content of CIK cells and proved they were all qualified. Then we transplanted the CIK cells to different patients for clinical trials.

\section{Genotyping analysis of clinical specimens}

As shown in Table 1, collected specimens were analyzed by genotyping; genotypes $\mathrm{A}$ and $\mathrm{D}$ are fewer proportion in patients; genotypes $\mathrm{B}$ and $\mathrm{C}$ are main proportion in patients.

\section{The changes of the liver indexes after CIK therapy}

The general tests for liver diseases detection is serum aminotransferase assays as the continuous enzyme concentration distribution in a population [31]. The marker of virus multiplication is serum hepatitis B virus (HBV) DNA level. In order to evaluate the CIK therapeutic effect in liver disease, three indexes were used, including ALT, AST and HBV DNA. After every patient was treated with $10^{10} \mathrm{CIK}$ cells for three times, the HBV DNA of the patients almost had a significant decrease by ten times (Fig. 2). The decrease of serum HBV DNA level in the patients suggest that the CIK therapy is effective to the patients with liver cancer. However, it is a strong risk predictor of hepatocellular carcinoma if the serum HBV DNA level is equal to or greater than 10,000 copies $/ \mathrm{mL}$ [32]. Although the HBV DNA level of the serum was decreased by CIK treatment, it was still higher than 10000 copies $/ \mathrm{mL}$, which suggests that the patients need further therapy. The ALT and AST levels were both decreased significantly after CIK therapy compared with untreated ones $(P<0.001)$ (Fig. 2$)$. The results once again demonstrated that by CIK cell therapy, the symptom of the liver disease was obviously relieved.

As shown in Fig. 3, the Pearson correlation coefficient of the liver indexes was calculated. The Pearson correlation coefficient of $\log (\mathrm{HBV}$ DNA), ALT and AST were 0.764, 0.364 and 0.340 . The results showed that CIK therapy can decrease the value of HBV DNA with little difference in patients. However, the ALT and AST had great differences among different patients after CIK therapy.

\section{The influence of CIK cells number on therapeutic consequents}

As shown in Table 2, no obvious HBV DNA copy number decreased as the number of CIK cells was lower than $10^{7}$ for every therapy. Notably, the HBV DNA copy number markedly decreased when the 

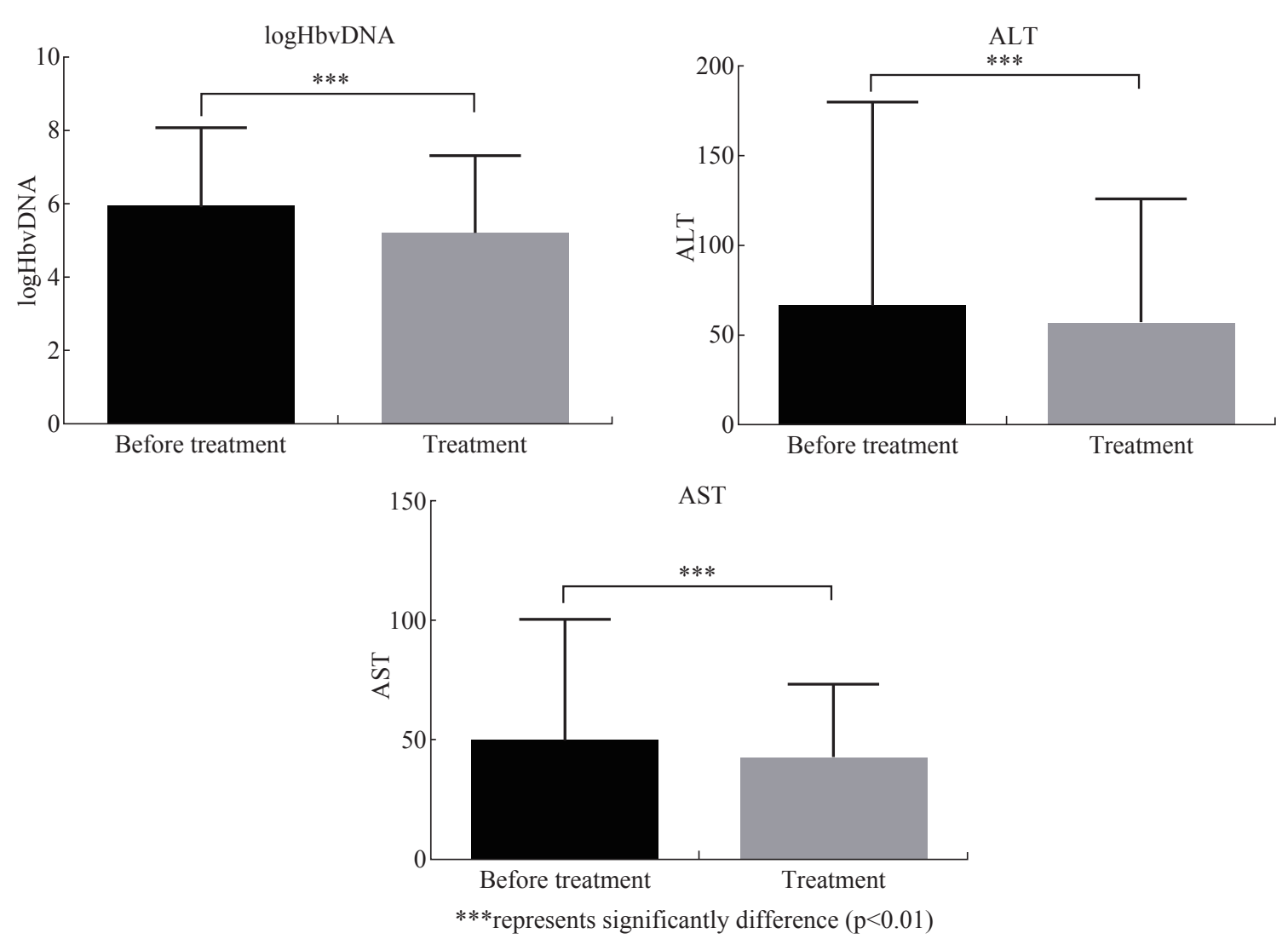

Fig. 2 The changes of the liver indexes after CIK therapy.
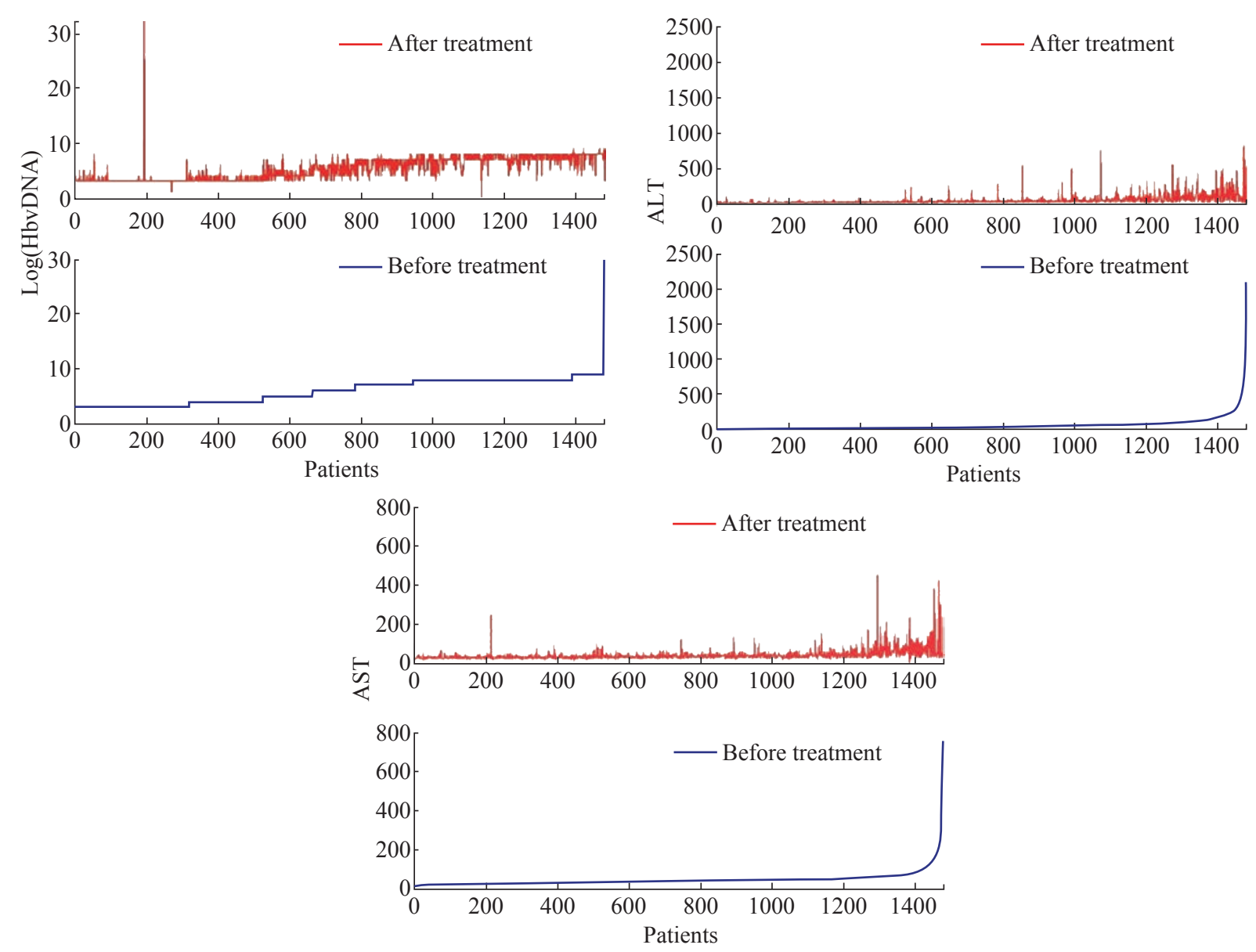

Fig. 3 The correlatioin of liver indexes after CIK therapy. 
number of CIK cells was more than $10^{10}$ for every therapy, which highly suggest that CIK cell number and quality can affect the therapeutic consequents.

\section{The influence of CIK treatment times on treatment effects}

As shown in Table 3, for different stages of HBV patients, including hepatitis patients, cirrhosis patients and hepatocellular cancer patients, as the treatment times increased, the HBV DNA copy number exhibited a gradual decreasing trend. After ten times of CIK cell therapy, the HBV DNA copy numbers decreased to several thousand copy number, which highly suggested that CIK treatment effects are closely associated with treatment times $(\mathrm{P}<0.01)$. Considering the clinical results, we suggest that CIK treatment times should be more than ten times in order to produce better clinical effects.

\section{The influence of HBV genotypes on CIK cell treatment efficacy}

As shown in Table 4, HBV DNA copy number and ALT as well as AST recovered to normal value scope after CIK cells were used ten times for hepatitis

Table 2 Effects of CIK cell numbers on HBV DNA copy numbers in sera of patients

\begin{tabular}{|c|c|c|c|}
\hline CIK cell number for three times & $\begin{array}{c}\text { Hepatitis patients } \\
\left(1,000, \text { copy number } \times 10^{5}\right)\end{array}$ & $\begin{array}{c}\text { Cirrhosis patients } \\
\left(400, \text { copy number } \times 10^{5}\right)\end{array}$ & $\begin{array}{l}\text { Liver cancer patients } \\
\left(80, \text { copy number } \times 10^{5}\right)\end{array}$ \\
\hline 0 & 6.523 & 7.6541 & 9.3417 \\
\hline $10^{5}$ & 5.2136 & 7.2396 & 9.2915 \\
\hline $10^{6}$ & 5.0147 & 7.2368 & 9.2167 \\
\hline $10^{7}$ & 5.0123 & 7.1349 & 9.0245 \\
\hline $10^{8}$ & 4.9986 & 7.0897 & 8.9573 \\
\hline $10^{9}$ & 3.2489 & 5.6431 & 6.4389 \\
\hline $10^{10}$ & 0.2034 & 0.20559 & 0.30453 \\
\hline
\end{tabular}

Table 3 Effects of CIK treatment times on HBV DNA copy number in sera of patients

\begin{tabular}{cccc}
\hline Treatment times & $\begin{array}{c}\text { Hepatitis patients } \\
\left(1,000, \text { copy number } \times 10^{5}\right)\end{array}$ & $\begin{array}{c}\text { Cirrhosis patients } \\
\left(400, \text { copy number } \times 10^{5}\right)\end{array}$ & $\begin{array}{c}\text { Liver cancer patients } \\
\left(80, \text { copy number } \times 10^{5}\right)\end{array}$ \\
\hline 0 & 6.523 & 7.6541 & 9.3417 \\
3 & 3.213 & 4.5678 & 8.2117 \\
6 & 1.765 & 3.7891 & 7.7893 \\
9 & 0.445 & 0.6573 & 0.0435 \\
10 & 0.02243 & 0.04533 & 0.07893 \\
12 & 0.01234 & 0.03395 & 0.05434 \\
16 & 0.01223 & 0.02371 & 0.02144 \\
\hline
\end{tabular}

Table 4 Effects of HBV genotypes on CIK therapy for hepatitis patients for ten times

\begin{tabular}{cccccc}
\hline Genotyping & Hepatitis patients & $\begin{array}{c}\text { HBV DNA } \\
\text { copy number }(<5,000)\end{array}$ & ALT (normal scope) & AST (normal scope) & Efficient ratio (\%) \\
\hline A & 150 & 135 & 135 & 135 & 90 \\
B & 350 & 263 & 263 & 263 & 75 \\
C & 410 & 258 & 258 & 258 & 63 \\
D & 90 & 83 & 83 & 83 & 92 \\
\hline
\end{tabular}


therapy with those HBV patients. Patients with genotypes $\mathrm{A}$ and $\mathrm{D}$ exhibited better therapeutic effects compared to patients with genotypes B and C, which indirectly suggests that HBV genotype may affect the CIK therapy consequence. Further investigation is under way.

From Table 5, we can see that for those patients with cirrhosis, HBV DNA copy number and ALT as well as AST also recovered to normal value scope after CIK cells were used for therapy for ten times. Patients with genotypes $\mathrm{A}$ and $\mathrm{D}$ exhibited better therapeutic effects compared to patients with genotypes $\mathrm{B}$ and $\mathrm{C}$, which once again confirms that HBV genotypes may affect the CIK therapy consequence of cirrhosis patients. Further experiments are under way.
As shown in Table 6, for those patients with hepatocellular cancer, after CIK cells were used for ten times of therapy, HBV DNA copy number and Alt as well as AST recovered to normal value scope. Patients with genotypes A and D showed better therapeutic effects compared to patients with genotypes B and C, which highly suggests that HBV genotypes may affect the CIK therapeutic consequence of hepatocellular cancer patients. Further supplementary experiments and functional studies are ongoing.

\section{Cytokine factor changes before and after treatment with CIK cells}

As shown in Fig. 4, for different stages of hepatitis patients, CIK cell therapy could increase cytokine

Table 5 Effects of HBV genotypes on CIK therapy for cirrhosis patients for ten times

\begin{tabular}{cccccc}
\hline Genotyping & Cirrhosis patients & $\begin{array}{c}\text { HBV DNA } \\
\text { copy number }(<5,000)\end{array}$ & ALT (normal scope) & AST (normal scope) & Efficient ratio (\%) \\
\hline A & 60 & 50 & 50 & 50 & 83 \\
B & 140 & 106 & 106 & 106 & 76 \\
C & 152 & 97 & 97 & 97 & 64 \\
D & 48 & 43 & 43 & 43 & 89 \\
\hline
\end{tabular}

Table 6 Effects of HBV genotypes on CIK therapy for hepatocellular cancer patients for ten times

\begin{tabular}{|c|c|c|c|c|c|c|}
\hline Genotyping & $\begin{array}{l}\text { Hepatocellular } \\
\text { cancer patients }\end{array}$ & $\begin{array}{l}\text { HBV DNA copy } \\
\text { number }(<5,000)\end{array}$ & ALT (normal scope) & AST (normal scope) & Lifespan (> 3years) & Efficient ratio(\%) \\
\hline A & 20 & 9 & 9 & 9 & 9 & 45 \\
\hline $\mathrm{B}$ & 30 & 9 & 9 & 9 & 9 & 30 \\
\hline $\mathrm{C}$ & 26 & 10 & 10 & 10 & 10 & 38 \\
\hline $\mathrm{D}$ & 4 & 3 & 3 & 3 & 3 & 75 \\
\hline
\end{tabular}

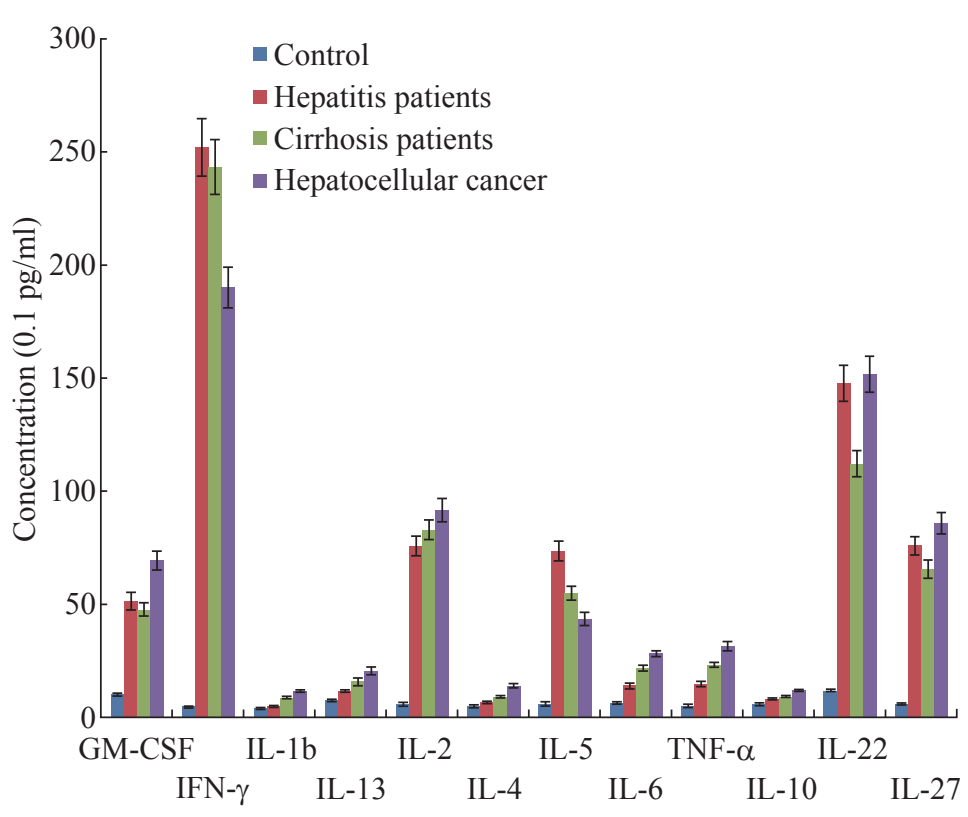

Fig. 4 Cytokine factor profile of different stages of hepatitis patients after CIK cell therapy for six times (CIK cell number for every treatment is more than $1 \times 10^{10}$ ). 
factor levels. For example, increased IFN-g and IL-2 concentration could enhance the efficacy of killing tumor cells and HBV. The cytokine factors such as IL4, IL-5, IL-6, IL-22 and IL-27 inhibit the proliferation of HBV and growth of liver cancer cells by regulating the immunological network. But the mechanism still needs further studying.

\section{Conclusions}

In summary, our clinical trial results show that CIK cell therapy can be used for different stages of HBV patients, including hepatitis patients, cirrhosis patients and hepatocellular cancer patients for its better therapeutic efficacy. CIK cell therapy effects are closely associated with CIK cell number and quality, and treatment times. Ten times of CIK cell therapy should be one treatment corner. Patients treated by more than ten times of CIK therapy obtained the good curative effect. The HBV genotypes were also associated with CIK cell therapeutic efficacy. Patients with HBV genotyes A and D exhibited better therapeutic effects than patients with genotypes B and $C$; the concrete molecular mechanism still needs further investigation. We also observed that cytokine factors such as GM-CSF, IFN-g, IL-1b, IL-2, IL4, IL-6, IL-10, IL-22 and IL-27 increased obviously, while their concrete mechanism still needs further studies. During the period of CIK treatment, all the patients felt better in sleep, diet and pain; the concrete mechanism is still underway. In conclusion, CIK cell therapy is a good alternative therapeutic method; it can be effectively used for treatment of different stages of HBV patients.

\section{Acknowledgments}

This work is supported by the National Key Scientific Projects for the Prevention and Control of HIV/AIDS and Viral Hepatitis of China (No. 2009ZX10004-311), Chinese 973 Project (No.2010CB933901 and 2015CB931802), National Natural Scientific Fund (No. 81225010, 81327002, and 31100717), 863 Project of China (2014AA020700), and Shanghai Science and Technology Fund (No. 13NM1401500 and 15DZ2252000).

\section{References}

[1] P. Marcellin, et al., Regression of cirrhosis during treatment with tenofovir disoproxil fumarate for chronic hepatitis B: A 5-year open-label follow-up study. The Lancet, 2013, 381(9865): 468-475.

[2] J. Lamontagne, J.C. Mell and M.J. Bouchard, Transcriptome-wide analysis of hepatitis B virus-mediated changes to normal hepatocyte gene expression. PLOS Pathog, 2016, 12(2): e1005438.

[3] T. Hosaka, et al., Long-term entecavir treatment reduces hepatocellular carcinoma incidence in patients with hepatitis B virus infection. Hepatology, 2013, 58(1): 98107.

[4] C.L. Lai, M.F. Yuen, Prevention of hepatitis B virusrelated hepatocellular carcinoma with antiviral therapy. Hepatology, 2013, 57(1): 399-408.

[5] G. Fattovich, Natural history and prognosis of hepatitis B. In Seminars in Liver Disease. Thieme Medical Publishers, Inc., 2003.

[6] E.A.F.T.S.O.T. Liver, EASL clinical practice guidelines: Management of chronic hepatitis B virus infection. Journal of hepatology, 2012, 57(1): 167-185.

[7] G. Raimondo, G. Cacciamo and C. Saitta, Hepatitis B virus and hepatitis $\mathrm{C}$ virus co-infection: additive players in chronic liver disease. Ann Hepatol, 2005, 4(2): 100106.

[8] W.D. Carey, The prevalence and natural history of hepatitis B in the 21 st century. Cleveland Clinic Journal of Medicine, 2009, 76: S2-5.

[9] J. Lucifora, et al., Specific and nonhepatotoxic degradation of nuclear hepatitis B virus cccDNA. Science, 2014, 343(6176): 1221-1228.

[10] C.J. Wooddell, et al., Hepatocyte-targeted RNAi therapeutics for the treatment of chronic hepatitis B virus infection. Molecular Therapy, 2013, 21(5): 973-985.

[11] S.C. Gordon, et al., Antiviral therapy for chronic hepatitis $B$ virus infection and development of hepatocellular carcinoma in a US population. Clinical Gastroenterology and Hepatology, 2014, 12(5): 885-893.

[12] R.E. Lanford, et al., GS-9620, an oral agonist of Tolllike receptor-7, induces prolonged suppression of hepatitis B virus in chronically infected chimpanzees. Gastroenterology, 2013, 144(7): 1508-1517.

[13] B. Rehermann, A. Bertoletti, Immunological aspects of antiviral therapy of chronic hepatitis B virus and hepatitis C virus infections. Hepatology, 2015, 61(2): 712-721.

[14] C. Trépo, H.L. Chan and A. Lok, Hepatitis B virus infection. The Lancet, 2014, 384(9959): 2053-2063.

[15] G. Schmidt-Wolf, R. Negrin, and I. Schmidt-Wolf, Activated $\mathrm{T}$ cells and cytokine-induced $\mathrm{CD}^{+}{ }^{+} \mathrm{CD} 56^{+}$ killer cells. Annals of Hematology, 1997, 74(2): 51-56.

[16] S.A. Rosenberg, J.C. Yang, and N.P. Restifo, Cancer immunotherapy: moving beyond current vaccines. Nature Medicine, 2004, 10(9): 909-915.

[17] K. Palucka, J. Banchereau, Cancer immunotherapy via dendritic cells. Nature Reviews Cancer, 2012, 12(4): 265277.

[18] I. Schmidt-Wolf, et al., Phenotypic characterization and identification of effector cells involved in tumor cell recognition of cytokine-induced killer cells. Experimental Hematology, 1993, 21(13): 1673-1679.

[19] R. Nen, P. Lu, Novel population of expanded human CD3, CD56 cells derived from T cells with potent in vivo antitumor activity in micewith severe combined immun0deficiency. J Immunol, 2004, 153(4): 1687-1696.

[20] A. Sasaki, et al., Preferential localization of human adherent lymphokine-activated killer cells in tumor microcirculation. Journal of the National Cancer Institute, 1991, 83(6): 433-437.

[21] I. Schmidt-Wolf, et al., Use of a SCID mouse/human lymphoma model to evaluate cytokine-induced killer cells with potent antitumor cell activity. The Journal of 
Experimental Medicine, 1991, 174(1): 139-149.

[22] R. Nishimura, et al., In vivo trafficking and survival of cytokine-induced killer cells resulting in minimal GVHD with retention of antitumor activity. Blood, 2008, 112(6): $2563-2574$

[23] A.A. Hombach, G. Rappl and H. Abken, Arming cytokine-induced killer cells with chimeric antigen receptors: CD28 outperforms combined CD28-OX40 "super-stimulation". Molecular Therapy, 2013, 21(12): 2268-2277.

[24] J. Zhang, et al., Human CIK cells loaded with gold nanoprisms as theranostic platform for targeted photoacoustic imaging and enhanced immunophotothermal combined therapy. Nano Biomed. Eng, 2016, 8(3): 109-124.

[25] Y. Yang, et al., Human CIK cells loaded with Au nanorods as a theranostic platform for targeted photoacoustic imaging and enhanced immunotherapy and photothermal therapy. Nanoscale Research Letters, 2016, 11(1): 1-13.

[26] G. Mesiano, et al., Cytokine-induced killer (CIK) cells as feasible and effective adoptive immunotherapy for the treatment of solid tumors. Expert Opinion on Biological Therapy, 2012, 12(6): 673-684

[27] D. Sangiolo, Cytokine induced killer cells as promising immunotherapy for solid tumors. J Cancer, 2011, 2(1): 363-368.

[28] G. Bonanno, et al., Thymoglobulin, interferon- $\gamma$ and interleukin-2 efficiently expand cytokine-induced killer (CIK) cells in clinical-grade cultures. Journal of Translational Medicine, 2010, 8(1): 1.

[29] A. Pievani, et al., Dual-functional capability of $\mathrm{CD}^{+}$ CD56 ${ }^{+}$CIK cells, a T-cell subset that acquires NK function and retains TCR-mediated specific cytotoxicity. Blood, 2011, 118(12): 3301-3310.

[30] C.-S. Wang, et al., Smoking and alanine aminotransferase levels in hepatitis $\mathrm{C}$ virus infection: implications for prevention of hepatitis $\mathrm{C}$ virus progression. Archives of Internal Medicine, 2002, 162(7): 811-815.

[31] C.K. Hyeon, et al., Normal serum aminotransferase concentration and risk of mortality from liver diseases: prospective cohort study. BMJ, 2004, 328(7446): 983.

[32] C.J. Chen, et al., Risk of hepatocellular carcinoma across a biological gradient of serum hepatitis B virus DNA level. Jama, 2006, 295(1): 65-73.

Copyright $@ 2016$ Fangfang Xia, Ruihua Song, Jingjing Zhang, Yang Zhu, Yao Yang, Ling He, Yun Cao, Chenlu Li, Zheng Wang, Shangli Cheng, Jian Ni, Lijun Ma, Ding Li and Daxiang Cui. This is an open-access article distributed under the terms of the Creative Commons Attribution License, which permits unrestricted use, distribution, and reproduction in any medium, provided the original author and source are credited. 